\title{
Percepciones del profesorado de primaria sobre las visitas educativas basadas en las Visual Thinking Strategies (VTS). DAFO de su aplicación en el Museo Picasso de Barcelona.
}

\author{
Myriam GONZÁLEZ-SANZ \\ Alex IBAÑEZ-ETXEBERRIA \\ Maria FELIU-TORRUELLA
}

Datos de contacto:

Myriam González-Sanz Universidad de Barcelona mgonzalezsanz@ub.edu

Alex Ibañez-Etxeberria Universidad del País

Vasco

alex.ibanez@ehu.eus

Maria Feliu-Torruella Universidad de Barcelona mfeliu@ub.edu

Recibido: 08/06/2021 Aceptado: 16/07/2021

\section{RESUMEN}

Se presenta un estudio de caso sobre la aplicación del método pedagógico Visual Thinking Strategies (VTS) a las visitas escolares del Museo Picasso de Barcelona. Teniendo como objetivo principal analizar las percepciones del profesorado de primaria y articular sus voces, se recurre a una metodología mixta a través de triangulaciones de instrumentos y técnicas de análisis. Para ello se opta por un análisis DAFO y se interrelacionan sus debilidades, amenazas, fortalezas y oportunidades mediante un mapa conceptual. Los docentes señalan muchos más aspectos positivos que negativos, concentrándose los primeros en tres áreas temáticas: los efectos cognitivos en el alumnado, el modelo educativo constructivista y significativo que impulsa y el concepto de museo como zona de confort donde el estudiantado puede participar y opinar libremente. Los aspectos negativos giran en torno a los rasgos propios del método, las características de la adaptación que de él hace la institución y las limitaciones estructurales del Museo Picasso. De acuerdo al profesorado, la implementación del método mejoraría al aplicarlo de manera más continuada y flexible, combinando las interpretaciones libres sobre obras de arte con contenido histórico-artístico. Al ser un método competencial se sugiere también un trabajo continuado en el museo y/o en la escuela.

PALABRAS CLAVE: Profesorado; Método de aprendizaje; Metodologías centradas en el alumnado; Arte; Museo. 


\title{
Teachers' perceptions of school programs based on the Visual Thinking Strategies (VTS): A SWOT analysis of the method's application at the Picasso Museum in Barcelona
}

\begin{abstract}
The article introduces a case study on the application of the Visual Thinking Strategies (VTS) teaching method to school activities at the Picasso Museum in Barcelona. From a mixed methodology, the perceptions of primary school teachers about their weaknesses, threats, strengths and opportunities are analyzed and articulated by means of a SWOT analysis, interrelating them through a conceptual map. Teachers point out many more positive than negative aspects, concentrating the former on three thematic areas: cognitive effects on students, the constructivist and meaningful educational model that it promotes, and the concept of the museum as a comfort zone where students can freely participate and give their opinion. The negative aspects revolve around the characteristics of the method, the features of the institution's adaptation of it, and the structural limitations of the Barcelona Picasso Museum. According to the teachers, the implementation of the method would improve when applying it in a more continuous and flexible way, combining free interpretations of works of art with historical-artistic content. Due to its skill-development approach, continued work in the museum and / or at school is also suggested.
\end{abstract}

KEYWORDS: Teachers; Teaching method; Student-centered methods; Art; Museum. 


\section{Introducción}

Durante décadas las escuelas han utilizado las visitas a museos de arte para fomentar el aprendizaje del alumnado (Luna e Ibañez-Etxeberria, 2020). Descubrir un nuevo contexto educativo y el contacto directo con obras auténticas aumenta su motivación y su interés por el arte y los museos (RK\&A, 2018). Sin embargo, no todas las actividades en museos impulsan un aprendizaje competencial ni significativo. Uno de los factores responsables son los métodos pedagógicos empleados.

El artículo analiza las percepciones del profesorado sobre uno de estos métodos: el Visual Thinking Strategies (en adelante VTS), en su aplicación a las actividades escolares del Museo Picasso de Barcelona (desde ahora MPB). El análisis se engloba en una investigación que recoge las perspectivas de todos los agentes participantes (e.g. González-Sanz et al. 2017).

\section{Metodologías pedagógicas en los museos de arte}

Desde los 80, la visión constructivista del aprendizaje como proceso activo ha llevado a muchos museos a implementar metodologías participativas basadas en el diálogo (Hein, 2006; Luna e Ibañez-Etxeberria, 2020). Primero, mediante métodos por indagación y, después, a través de estrategias dialógicas más abiertas en las que contenidos y preguntas vienen marcados por los intereses del alumnado y no por los de los educadores.

Estas metodologías dialógicas, que Hubard califica de no predeterminadas (2015), responden a la redefinición de tres conceptos: a) se basan en una educación reformulada por las pedagogías críticas (Freire, 2005), que defienden métodos más horizontales y democráticos que estimulen el pensamiento crítico y respeten los derechos de los estudiantes como ciudadanos (Mörsch, 2009; Siddiqui et al., 2019); b) se relacionan con el aprendizaje en el museo como proceso individual y colectivo mediante el cual los visitantes crean sus propios significados a través de sus conocimientos y experiencias previas (Falk y Dierking, 2000; Hein, 2006; HooperGreenhill, 2000); c) se inscriben en el nuevo paradigma del museo como espacio para el diálogo y la interacción comunitaria (e.g., Simon, 2010), en cuyos programas muchos autores afines a las teorías feminista y decolonial (e.g. Dewhurst y Kendrick, 2016; Mörsch, 2009) reclaman que se impulse la deconstrucción crítica de las interpretaciones blancas y heteropatriarcales del arte, resignificándolas a través de debates.

A pesar de estas redefiniciones y aunque Housen (1999) advertía sobre la inadecuación de las metodologías discursivas para los espectadores con menos experiencia en la interacción con el arte, Mörsch denunciaba en 2009 el predominio de prácticas transmisivas (Hooper-Greenhill, 2000); en estas, el educador difunde el discurso reproductivo de su institución al público escolar, manteniendo un rol privilegiado como experto y emisor principal de contenidos mientras la participación del alumnado se restringe. Un diagnóstico que Arriaga subscribía respecto a España en 2010. 
Y es que, aunque muchos métodos dialógicos siguen los nuevos modelos educativos competenciales, aquellos que otorgan la autoridad interpretativa al espectador generan polémica. Bien porque se considera que el museo no debe renunciar a su responsabilidad como productor y difusor de significados (Meszaros, 2006), porque se cree que no aportar contenido contextual empobrece las interpretaciones individuales (Arriaga, 2010; López y Kivatinetz, 2006) o porque se piensa que no generan una relectura crítica de las obras (Lechuga-Jiménez, 2018). La polémica es particularmente marcada en relación al método VTS, descrito seguidamente.

\section{Una breve historia del VTS y sus evaluaciones}

Según Housen (1999) y Yenawine el VTS es un método pedagógico que aspira a facilitar el aprendizaje a través del arte, desarrollando la alfabetización visual, las capacidades comunicativas y el pensamiento crítico y creativo de sus usuarios (Hailey et al, 2015). Con estos objetivos busca generar un espacio seguro o de confort (Ritchhart, 2007) en el que se invita al alumnado a observar pausadamente (slow looking) y a analizar, deducir, compartir y argumentar sus propias interpretaciones de varias obras de arte; un entorno de diálogo respetuoso que aspira a fomentar la tolerancia hacia otras opiniones y transmitir que el arte tiene muchos significados posibles. Para ello los educadores VTS deben facilitar un diálogo sobre cada pieza utilizando tres preguntas abiertas: ¿Qué (crees que) está pasando en esta obra? ¿Qué ves en la obra que te hace pensar eso? ¿Qué más podemos encontrar? Al basarse en que en el arte no existe una única verdad, y para motivar que los participantes compartan sus teorías sin miedo al error, los educadores aceptan todas aquellas lecturas justificadas mediante el análisis formal de las obras. Además, parafrasean cada comentario de los estudiantes para validar sus perspectivas y enriquecer su vocabulario.

Creado como Visual Thinking Curriculum (VTC) en los 80 en el MoMA y orientado a museos y a escuelas, el método llegó a España como VTS a finales de los 90, siendo implementado en el programa Mira! (Fundación La Caixa, 1998-2002) (Pou, 2002), el Museo Picasso de Málaga o el Centro Atlántico de Arte Moderno (CAAM) (Paunero, 2018), entre otros. A pesar de las críticas de un sector de académicos (e.g. Arriaga, 2010; López y Kivatinetz, 2006), en los últimos años se ha detectado un interés renovado en los museos de Cataluña.

Desgraciadamente las evaluaciones de su uso en actividades escolares en museos son escasas (Hailey et al, 2015), analizando la mayoría programas multivisita o desarrollados principalmente en la escuela. Entre ellas destacan la de aplicación del VTC al MoMA (Tishman et al, 1999), la del Mira! (Pou, 2002) y las del programa Thinking through Art del Isabella Stewart Gardner Museum (e.g. Adams et al, 2007; Burchenal y Grohe, 2007). Todas muestran un aumento moderado pero significativo de las habilidades de pensamiento crítico y expresión oral de los participantes, de su aceptación de la subjetividad interpretativa del arte y de su predisposición a la escucha y al diálogo respetuoso. En muchos casos, se registra también un interés creciente en el arte y en los museos (e.g. Kisida et al, 2017) y un incremento de autoestima y 
autoconfianza, debido a que los estudiantes comprenden que pueden intervenir sin temor a equivocarse.

Sin embargo, estos estudios corresponden a programas a medio y largo plazo que implican múltiples sesiones en el museo y/o la escuela, mientras que el presente análisis gira en torno a las percepciones sobre actividades monovisita. En ese sentido, las evaluaciones de referencia serían las de Kisida, Bowen y Greene (e.g. 2016, 2017) respecto a una visita VTS al Crystal Museum (EEUU), y el informe de RK\&A (2018) en torno a 100 actividades de museos estadounidenses basadas en metodologías constructivistas (sin ser todas VTS). Las conclusiones de estos estudios coinciden con las de los programas multivisita, si bien los efectos son más modestos, remarcándose la dificultad de establecer relaciones de causalidad entre actividades y efectos.

\section{Percepciones del profesorado acerca de las visitas escolares a museos y sus metodologías pedagógicas}

Más allá de las controversias entre académicos y gestores, subrayamos con Huerta et al (2014) que para implementar nuevas metodologías es necesario contar con la voluntad del profesorado. De aquí que resulte fundamental analizar y articular sus percepciones. Aunque en los últimos años los equipos de Calaf, Fontal (e.g. GómezRedondo et al, 2017) y la Comunitat d'Aprenentatge Patrimoni i Escola (en adelante, CoP) (2018) han desarrollado criterios e indicadores evaluativos de las actividades educativas patrimoniales, y más allá del corpus sobre maestras y museos de Huerta (e.g. 2009) o Escribano-Miralles (e.g. 2019), son pocas las evaluaciones directas de docentes en activo sobre estrategias pedagógicas empleadas en museos de arte.

Retomamos así algunas investigaciones que han examinado sus expectativas, objetivos y percepciones sobre las actividades escolares de diferentes tipos de museo (e.g. Anderson et al, 2006; RK\&A 2018; Viladot, 2015), descartando aquellas enfocadas en las opiniones de docentes en formación. Más allá de la tipología de museo, el diagnóstico se repite: una de las finalidades principales del profesorado al organizar visitas didácticas es tratar contenidos del currículum (e.g. Marcus et al, 2012; Loran, 2014; Viladot, 2015). Simultáneamente, muchos parecen valorar las estrategias disruptivas (Loran, 2014; Marcus et al, 2012) y demandan enfoques participativos y discusiones activas que fomenten el pensamiento crítico y creativo (RK\&A, 2018; Viladot, 2015).

Aún así, las técnicas dialógicas que confieren la autoridad interpretativa al alumnado y /o renuncian a aportar contenido experto, como el VTS, también generan controversia en el profesorado. Aunque no se ha podido acceder a investigaciones sistemáticas desde la perspectiva docente sobre programas escolares VTS en museos, la literatura científica lo evidencia. Mientras que algunos se entusiasman al descubrir un método democratizador que refuerza las habilidades de pensamiento y comunicación (e.g. Burchenal y Grohe, 2007; Pou, 2002), otros critican el desconocimiento de sus dinámicas y objetivos, la desconexión con las estrategias y contenidos del currículum formal (Tishman et al, 1999; Pou, 2002), la no introducción 
de nuevo contenido histórico-artístico por los educadores y al rechazo a corregir las interpretaciones libres de los niños. (e.g. Cappello y Walker, 2016; Paunero, 2018).

Para comprender estas discrepancias es importante contemplar factores como el tipo de formación previa sobre arte de los docentes. Arriaga (2010), Huerta et al, (2014) y Tishman et al. (1999) sugieren que las experiencias pasadas de los docentes al aprender arte y sus concepciones sobre qué es y cómo debe ser enseñado son aspectos clave para entender las valoraciones que hacen de los programas y las metodologías educativas museísticas. Aquellos que aprendieron mediante un modelo tradicional, en el que la obra se asocia a una única verdad y a mucho contenido contextual, suelen tener dificultades en aceptar que el arte es un lenguaje abierto a múltiples interpretaciones, donde el significado es completado por el observador (Hein, 2007). Una solución puede ser inspirarse en propuestas basadas en métodos similares al VTS como las Thinking Routines de Project Zero (Ritchhart, 2007; Trouli, 2020), que permiten la coexistencia de dos tipos de contenidos: los del museo y los que construyen sus visitantes.

Cappello y Walker (2016) aportan otro factor al afirmar que las preocupaciones de los docentes sobre el VTS aparecen solo en contextos formales, donde les cuesta asumir que todas las respuestas puedan ser válidas. Sus resultados indican que el profesorado considera los museos como un entorno más adecuado para el VTS, al ser espacios de aprendizaje más libres con menor presión por alcanzar objetivos y contenidos curriculares.

A esto deben sumarse aspectos generales reiterados en la literatura científica: la actual obsesión por contabilizar resultados en educación (Hunter-Doniger y Herring, 2017); las limitaciones de tiempo y presupuesto; las dificultades logísticas al preparar visitas a museos (e.g. Loran, 2014; Marcus et al, 2012), y la experiencia de los docentes con metodologías activas y participativas. Así como su grado de acuerdo con las citadas revisiones de los conceptos educación, museo y aprendizaje en el museo.

Frente a las discrepancias evidenciadas en la revisión de la literatura, el objetivo de este artículo es analizar las percepciones del profesorado de primaria sobre la adaptación del VTS a las actividades escolares en el MPB y establecer cuáles son las debilidades, amenazas, fortalezas y oportunidades que detectan en ella para mejorarla en base a estas.

\section{Método}

Para responder a este objetivo se optó por una metodología mixta mediante triangulaciones de instrumentos y técnicas de análisis, que facilitase la comprensión de un fenómeno que se podría calificar de complejo. En concordancia con la metodología mixta utilizada se optó por el método de encuesta o de interrogación y por el método observacional, recayendo el mayor peso en aquellos aspectos más cualitativos. Esta decisión vino dada por las circunstancias extraordinarias del MPB cuando se inició el trabajo de campo (2015/16), puesto que entonces era el único museo de la ciudad que ofrecía al profesorado única y exclusivamente actividades escolares basadas en el VTS. El que una de las investigadoras trabajara entonces como educadora en el MPB garantizó el acceso al campo y al profesorado, entre el cual se 
había generado controversia debido al desconocimiento del método, al cambio respecto a un modelo anterior donde el contenido contextual tenía un peso muy importante, y a la inexistencia de alternativas metodológicas.

\section{Población y muestra}

El MPB era entonces uno de los más visitados de la ciudad, sumándose una gran afluencia de público turista a las numerosas visitas escolares. Debido al número de estas últimas, la población se delimitó al profesorado acompañante de aquellos grupos de 5o y 6 de primaria con reserva para el 2017. La participación requería un esfuerzo importante, de aquí que se optara por un muestreo no probabilístico por voluntariado. Tras garantizar el anonimato y la confidencialidad de los datos, se obtuvo el consentimiento verbal informado por parte de 12 escuelas de municipios de Cataluña. El día de su visita al museo los docentes recibieron un cuestionario, que debían rellenar en 15 días. Finalmente se alcanzó una muestra viva de 22 profesionales.

\section{Instrumento}

El cuestionario docente (CD) se componía de 18 ítems, 10 de respuesta abierta y 8 de respuesta cerrada. Para garantizar su validez se aseguró la adecuación de los ítems a las dimensiones e indicadores extraídas del marco teórico, a la que se sumó el juicio de expertos y sucesivos pilotajes. Paralelamente, se buscó la validez de contenidos a través de la triangulación de las fuentes y las técnicas de análisis.

\section{Tratamiento y análisis de los datos}

Puesto que el CD no se diseñó originalmente para efectuar un DAFO, se transcribieron y revisaron todos sus ítems, analizándose los más relevantes (ver Anexo I). Los datos cuantitativos se trataron a través de SPSS 25.0, llevándose a cabo análisis descriptivos y comparativos. Los datos cualitativos se redujeron y categorizaron desde procedimientos deductivos e inductivos hasta la saturación teórica de las categorías (Miles et al., 2014) mediante NVIVO 12. Las categorías a priori se reformularon y completaron con otras nuevas, validándolas teóricamente a través del contraste con expertos hasta configurar los libros de códigos finales. Finalmente, los resultados se triangularon hasta la obtención de un DAFO y de un mapa conceptual que permitieron comprenderlos mejor.

El procedimiento de análisis DAFO permite mostrar sintéticamente "las limitaciones y potencialidades que se vislumbran desde y respecto a la organización o colectivo estudiado", dividiéndolos en factores positivos y negativos, internos (fortalezas y debilidades) y externos (amenazas y oportunidades) (Gil-Zafra, 2001:127). Su aplicación en museos (Martín-Cáceres y Cuenca-López, 2016; Castejón, 2019) posibilita efectuar diagnósticos y propuestas de optimización, sumando la perspectiva de los participantes a la de los investigadores. En su diseño se tuvieron en 
cuenta las categorías más relevantes, buscando su coherencia y la de su clasificación en áreas temáticas para su posterior triangulación. (Ver definiciones en el Anexo II).

Siempre que se creyó conveniente, se contrastaron los resultados del CD con una triangulación por complementariedad con la observación no participante (ONP) de las actividades escolares efectuada durante 2017.

\section{Resultados y discusión}

El diagrama de jerarquía (véase Figura 1) obtenido mediante el análisis de contenido evidencia la percepción positiva de las actividades por parte del profesorado, que otorga un enorme peso a los puntos fuertes frente a los débiles. Los aspectos positivos más citados aluden a los efectos cognitivos en el alumnado y a un modelo de aprendizaje constructivista y significativo que pone al estudiantado en el centro, pero también a factores ligados al museo como zona de confort para participar, opinar y debatir libremente. Entre los aspectos negativos remarcan aquellos relacionados, bien con el propio método VTS, bien con la adaptación que de él hace el MPB en sus actividades escolares, o bien con las limitaciones estructurales del propio museo.

\section{Figura 1}

Diagrama de jerarquía de los puntos fuertes y débiles detectados por el profesorado. Fuente: elaboración propia.

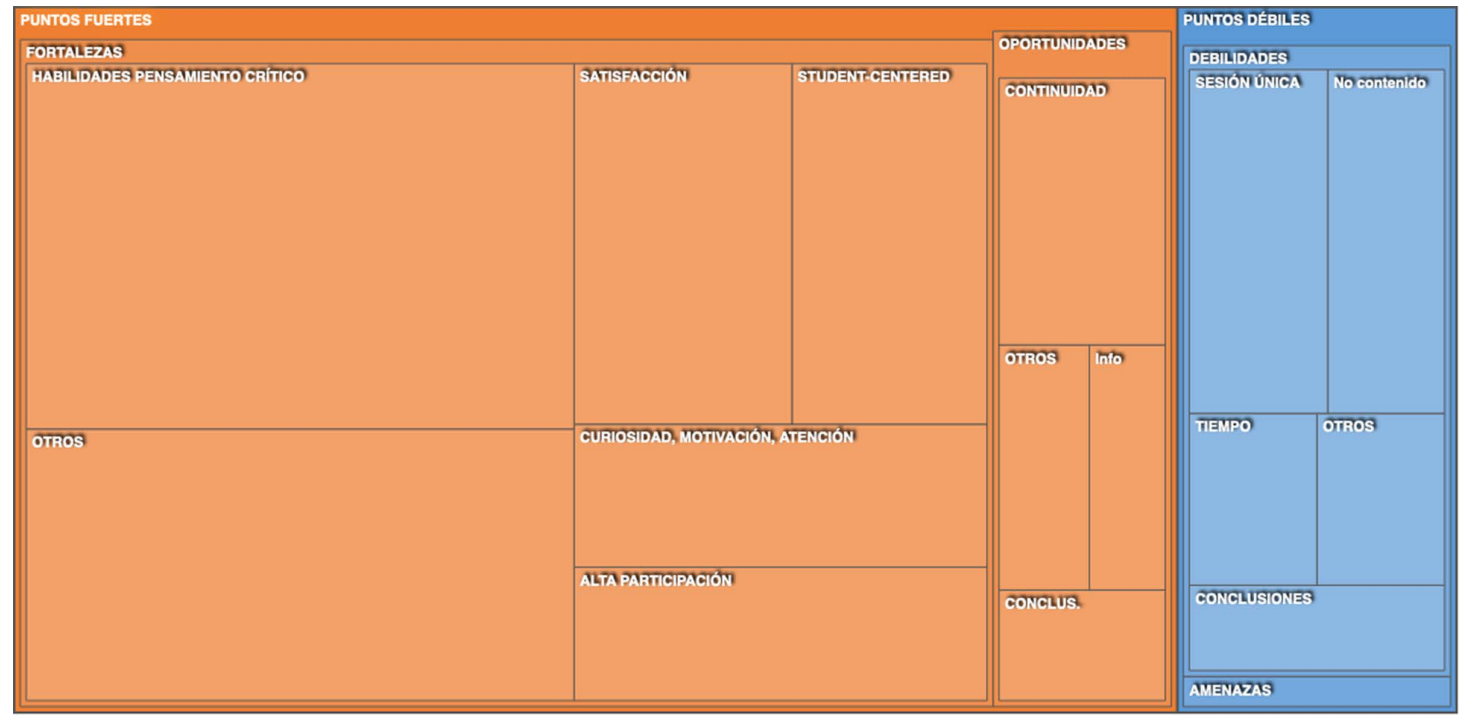

El DAFO (ver Figura 2) muestra que los docentes concentran sus reflexiones en los aspectos internos y presentes (fortalezas y debilidades), detectando menos oportunidades y muy pocas amenazas. 


\section{Figura 2}

DAFO del profesorado. Fuente: elaboración propia.

\begin{tabular}{|c|c|}
\hline DEBILIDADES & AMENAZAS \\
\hline $\begin{array}{l}\text { Falta de explicaciones con contenidos } \\
\text { histórico-artísticos } \\
\text { Aplicación en una única sesión } \\
\text { El apartado de conclusiones es insuficiente } \\
\text { Polarización sobre la duración y distribución } \\
\text { del tiempo de la actividad } \\
\text { Otros (<10\%) } \\
\circ \quad \text { Limitaciones estructurales del MPB } \\
\text { Grupos demasiado grandes } \\
\text { Aceptación de todas las respuestas } \\
\text { sin objetividad }\end{array}$ & $\begin{array}{l}\text { El aprovechamiento de la actividad } \\
\text { depende de los saberes que este traiga } \\
\text { consigo }\end{array}$ \\
\hline FORTALEZAS & OPORTUNIDADES \\
\hline $\begin{array}{l}\text { Desarrollo de habilidades ligadas al } \\
\text { pensamiento crítico } \\
\text { Pone al alumnado en el centro: papel activo, } \\
\text { autonomía y protagonismo } \\
\text { - Fomento curiosidad/motivación/atención } \\
\text { - La alta participación que potencia } \\
\text { - Satisfacción del alumnado } \\
\text { Otros (<10\%) } \\
\circ \quad \text { Desarrollo habilidades expresión oral } \\
\circ \quad \begin{array}{l}\text { Integración y reivindicación de todo } \\
\text { el alumnado }\end{array} \\
\text { Alfabetización visual } \\
\circ \quad \begin{array}{l}\text { Desarrollo de una nueva mirada y } \\
\text { sensibilidad hacia el arte }\end{array} \\
\text { Posibilita vínculos con experiencias y } \\
\text { conocimientos previos } \\
\text { Aprendizaje colaborativo }\end{array}$ & 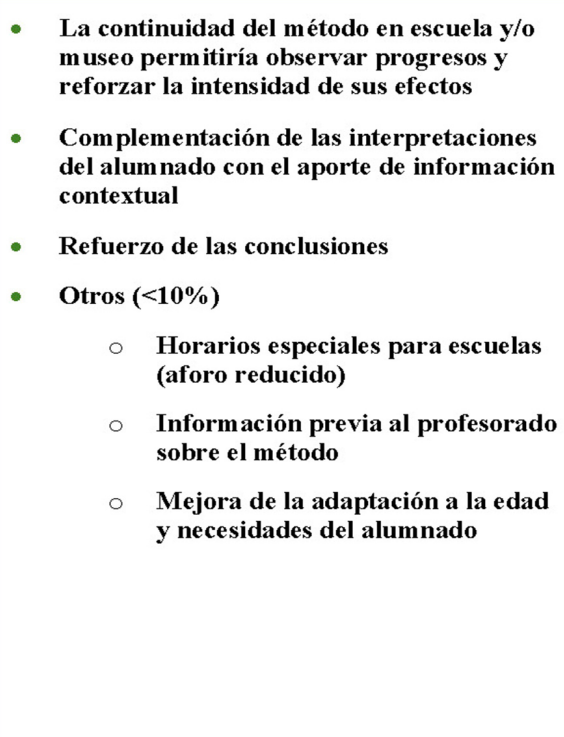 \\
\hline
\end{tabular}

\section{Puntos fuertes}

\section{Fortalezas}

Se focalizan en tres áreas temáticas: los efectos en el desarrollo cognitivo y competencial del alumnado, el modelo de aprendizaje centrado en los estudiantes que promulga, y la configuración del museo como entorno de aprendizaje seguro en el que participar y debatir sin miedo al error. 
Efectos cognitivos en el alumnado

Los docentes remarcan principalmente que ayuda a desarrollar las habilidades ligadas al pensamiento crítico, las capacidades de expresión oral y la alfabetización visual, todos ellos objetivos explícitos del VTS (Hailey et al, 2015). Entre las primeras citan, por este orden, la observación atenta (slow looking), la reflexión, la capacidad de análisis y deducción y la de argumentación.

"Ayudar a los alumnos a razonar, deducir, y conectar ideas a través de la observación atenta de obras de arte." (Docente 20)

También señalan que contribuye a desarrollar habilidades de expresión oral, si bien su relevancia es mucho menor que la de las capacidades anteriores, como suele suceder en las actividades monovisita. Aparecen después la alfabetización visual, entendida como el estímulo de la autonomía frente a la obra más allá de sus conocimientos sobre ella y otra categoría relacionada, el desarrollo de nueva mirada y sensibilidad hacia el arte.

"Aprender a hacerse preguntas sobre la obra de arte y a saber mirar." (Docente 16)

Las fortalezas de esta área coinciden con las evaluaciones ya citadas, si bien la percepción sobre las dos primeras se acerca más a los efectos de los programas a largo plazo (e.g. Adams et al, 2007; Pou, 2002) que a los monovisita (Kisida et al, 2017; RA\&K, 2018).

Aprendizaje activo y significativo centrado en el alumnado

El profesorado destaca muchos de los factores ligados al aprendizaje constructivista y significativo, reconociendo enseguida el enfoque student-centered y valorando el cambio de los roles tradicionales de educadores y alumnado. El poner al alumnado en el centro y otorgarle un papel activo, autonomía y protagonismo, un aspecto diferencial del VTS como método educativo, es la segunda fortaleza mas apuntada.

"Me parece genial la iniciativa de poner el niño en el centro del aprendizaje. ;Buen trabajo! (Docente 18)

Los resultados subrayan las conclusiones de Burchenal y Grohe (2007) y Pou (2002) y contradicen las del sector crítico (e.g. Arriaga, 2010; López y Kitavinetz, 2006) cuando afirman que el VTS es una manera encubierta de mantener al educador como protagonista de un proceso educativo transmisivo y unidireccional. Sin embargo, aunque muchos docentes critican la no transmisión de contenidos contextuales, ninguno afirma que la actividad parte de los intereses del alumnado. Esto sugiere que no tienen la capacidad de decisión sobre los temas a tratar de las metodologías dialógicas abiertas descritas por Hubard (2015).

Íntimamente relacionado, le sigue el estímulo de la motivación y la curiosidad y el mantenimiento de la atención, en línea con evaluaciones previas (e.g. Tishman et al, 1999). Aún así, algún docente marcaba como debilidad que tienen dificultades para mantener la concentración durante los 10-12 minutos dedicados a la observación de cada obra.

“Además, les piden que interactúen, pidiendo su opinión y percepciones, lo convierte en una actividad más motivadora." (Docente 11) 
Aunque su incidencia es menor, cinco de los 22 participantes destacan que posibilita vínculos con los conocimientos previos del alumnado, un aspecto singular del aprendizaje significativo (Ordóñez y Mohedano, 2018).

"Permite extraer conclusiones personales en base a los conocimientos y experiencias propias.» (Docente 18)

Finalmente, aunque solo un docente alude espontáneamente a la satisfacción que la actividad provoca en el alumnado, cuando se les pide que valoren este aspecto (ver ítem 7 en anexo I) lo hacen con una media de un 4,2 sobre 5, siendo uno de los más destacados.

El museo como zona de confort para participar, opinar y debatir libremente

Un conjunto de categorías puede agruparse en torno a una idea que el VTS comparte con teóricos ligados a otras rutinas de pensamiento (e.g. Ritchhart, 2007) y con autores asociados al nuevo paradigma de museo (e. g. Mörsch, 2009; Simon, 2010): convertir el museo en un espacio seguro donde los participantes puedan opinar y debatir de manera respetuosa, construyendo conocimiento entre iguales. El no sentirse juzgados, puesto que todas las interpretaciones son válidas si se justifican mediante el análisis formal, fomenta la participación -la cuarta fortaleza del DAFO- e inculca el respeto a la diversidad de opiniones, estimulando el aprendizaje colaborativo.

Además, relacionan este incremento participativo con la integración $\mathbf{y}$ reivindicación de todos los estudiantes durante el proceso, sea porque el VTS se adapta a las características del alumnado o porque saca a la luz las capacidades de los que pasan desapercibidos en clase (Paunero, 2018).

"Que alumnos que normalmente no hacen comentarios demasiado "acertados", allí estuvieron muy correctos." (Docente 9)

Es interesante remarcar que el profesorado destaca los efectos que provoca en el alumnado que todas las aportaciones sean valoradas por los educadores, como que se genere una mayor interacción y que todos pueden opinar libremente. En cambio, en muchas evaluaciones previas se pone el acento en que esta estrategia desarrolla la comprensión de los estudiantes sobre la subjetividad interpretativa del arte y su consiguiente respeto hacia las opiniones de sus compañeros (e.g. Tishman et al. 1999; RK\&A, 2018).

\section{Oportunidades}

La mitad del profesorado subraya como oportunidad la continuidad de trabajo con el método en la escuela o el museo permitiría reforzar sus efectos y observar progresos. El enfoque competencial del VTS requiere tiempo, de aquí que dos docentes propongan aumentar las sesiones en el museo, en la línea de programas como el Thinking through Art (Burchenal y Grohe, 2007):

"Quizás se podrían hacer 2 actividades, una al inicio de curso y otra al final en el museo, y en medio un trabajo en el centro con los maestros." (Docente 11)

La segunda oportunidad más señalada es complementar las interpretaciones del alumnado con el aporte de información contextual, combinando las ventajas de 
ambas maneras de entender la educación a través del arte. Al sugerir este enfoque mixto, los participantes se acercan a las adaptaciones del VTS que se han ido dando en museos pioneros en su aplicación como el MoMA y el Museo Picasso de Málaga. En ellos, el uso "puro" del método se ha substituido por una alternancia entre rondas de interpretaciones libres con otras de aportación de contenido, que ayuda a profundizar en la observación y el diálogo sobre las obras.

La tercera oportunidad está ligada a la anterior y a la debilidad el apartado de conclusiones es insuficiente. Se sugiere que deben reforzarse, introduciéndose contenidos histórico-artísticos esenciales, sobre todo.

"Se debería concluir la actividad como mínimo haciendo saber a los alumnos: nombre de la obra, situación de la obra, explicación breve del por qué la hizo el autor."

(Docente 21)

Uno de los participantes alude a que debe utilizarse las conclusiones para "hacer consciente a los niños de los que han aprendido, cómo y porqué" (Docente 18). Algo sorprendente, ya que la ONP confirma la importancia que los educadores dan a desarrollar esta reflexión sobre el proceso de aprendizaje en la implementación del MPB.

Entre las oportunidades menores sobresale la apuesta por ofrecer las actividades escolares en horarios de menor afluencia de público o a puerta cerrada. Además, dos participantes proponen que se informe previamente al profesorado sobre el método, evidenciando que, pese a la importancia de la formación por parte de los museos (Huerta et al, 2014; Huerta, 2009), la comunicación museo-profesor no siempre funciona y que algunos desconocen las frecuentes sesiones formativas ofrecidas por el MPB. Finalmente, la petición de dos participantes de mejorar la adaptación a la edad y necesidades del alumnado, contrasta con la filosofía inclusiva del método (Housen, 1999) y con los resultados de Kisida et al (2016) y Tishman et al (1999), entre otros.

\section{Puntos débiles}

Se considera muy significativo que 6 de los 22 docentes no señalen ningún punto débil cuando se les pregunta directamente. Así mismo, destaca la desproporción entre los aspectos negativos actuales y bajo control del MPB, y los externos y potenciales.

\section{Debilidades}

Se estructuran en torno a tres grandes temas: aspectos referentes al método VTS en sí, factores relativos a la adaptación que de él hace el MPB en su oferta escolar y limitaciones del propio museo.

Relacionadas con aspectos propios del método VTS

Una de las dos debilidades más remarcadas es la falta de explicaciones con contenidos histórico-artísticos por parte de los educadores. Aunque muchos docentes señalan como fortalezas que el VTS de protagonismo al alumnado y fomente que interpreten la obra de manera autónoma, la mitad continúa rechazando que no se 
introduzca información contextual. Coinciden así con las conclusiones de Pou (2002), Tishman et al (1999), Cappello y Walker (2016) y Burchenal y Grohe (2007).

"Quizás, me ha faltado que los guías contrastasen la opinión de los alumnos con la historia real de los cuadros" (Docente 20)

Este último testimonio, en línea con otro que rechaza que se acepten todas las respuestas sin objetividad, evidencia que en un sector del profesorado pervive la visión clásica según la cual solo hay una lectura "real" de cada obra de arte. Enlaza además con las teorías de Meszaros (2006) y Arriaga (2010) sobre como, al renunciar a su autoridad interpretativa, el museo elimina una de las pocas oportunidades de la escuela para recibir información especializada y empobrece el proceso de construcción de significados del alumnado. Sorprendentemente, aunque la ONP mostró que los educadores introducen o promueven la aparición de ciertos contenidos que permiten conectar la visita con el posterior taller, los docentes no aluden a esta combinación de estrategias propia de la adaptación del MPB.

Una debilidad relacionada con la anterior es que el apartado de conclusiones es insuficiente y que debería utilizarse para incorporar contenidos histórico-artísticos, evidenciar más las ideas a las que se ha llegado como grupo o concienciarles más del propio proceso de aprendizaje, propuestas todas ellas recogidas en oportunidades.

\section{Relacionadas con la implementación del método en el MPB}

La segunda categoría en relevancia es que un método de enfoque competencial como el VTS se aplique en una sola sesión. Aunque no ha sido señalada por los docentes de forma espontánea, 11 participantes reconocen la debilidad al ser preguntados (ver ítem 14 en el anexo I): 4 afirman tajantemente que es insuficiente, mientras que 7 consideran que los progresos y la intensidad de los efectos dependen de la continuidad. Algo que confirma la comparación entre las evaluaciones longitudinales (e.g Pou, 2002) y las de las actividades monovisita (e.g. Kisida et al. 2016).

Una limitación difícil de superar es la polarización del profesorado sobre cuál debería ser la duración y distribución del tiempo de la actividad, ya que unos consideran que el tiempo dedicado al análisis de cada obra o a la visita es corto y otros lo ven demasiado largo.

Finalmente, dos de ellos apuntan que los grupos de 25 estudiantes del MPB son demasiado grandes para conseguir la participación de todos. Esto conecta con la división de las clases en dos grupos de máximo 15 que el MoMA impulsó hace unos años y que aplican también otros museos estadounidenses con metodologías dialógicas.

\section{Limitaciones estructurales del MPB}

Teniendo en cuenta que el MPB es uno de los museos más visitados de Cataluña asombra que solo tres docentes aludan a las limitaciones del museo como espacio. En concreto, consideran que el ruido y el exceso de aforo permitido dificultan el seguimiento de la actividad y proponen oportunidades de mejora. 


\section{Amenazas}

Los docentes apenas detectan aspectos negativos que no sean internos y actuales. Solo dos señalan un par de limitaciones potenciales: que el aprovechamiento de la actividad depende de los saberes que traiga consigo el alumnado y que la timidez de ciertos estudiantes retrae su participación.

Como se observa seguidamente en el mapa conceptual (Figura 3), estas amenazas representan el reverso de características del VTS que gran parte del profesorado destaca como fortalezas principales (papel activo y protagonista del alumnado y la alta participación que potencia).

\section{Figura 3}

Mapa conceptual con las relaciones entre categorías de los cuatro aspectos del DAFO del profesorado. Fuente: elaboración propia

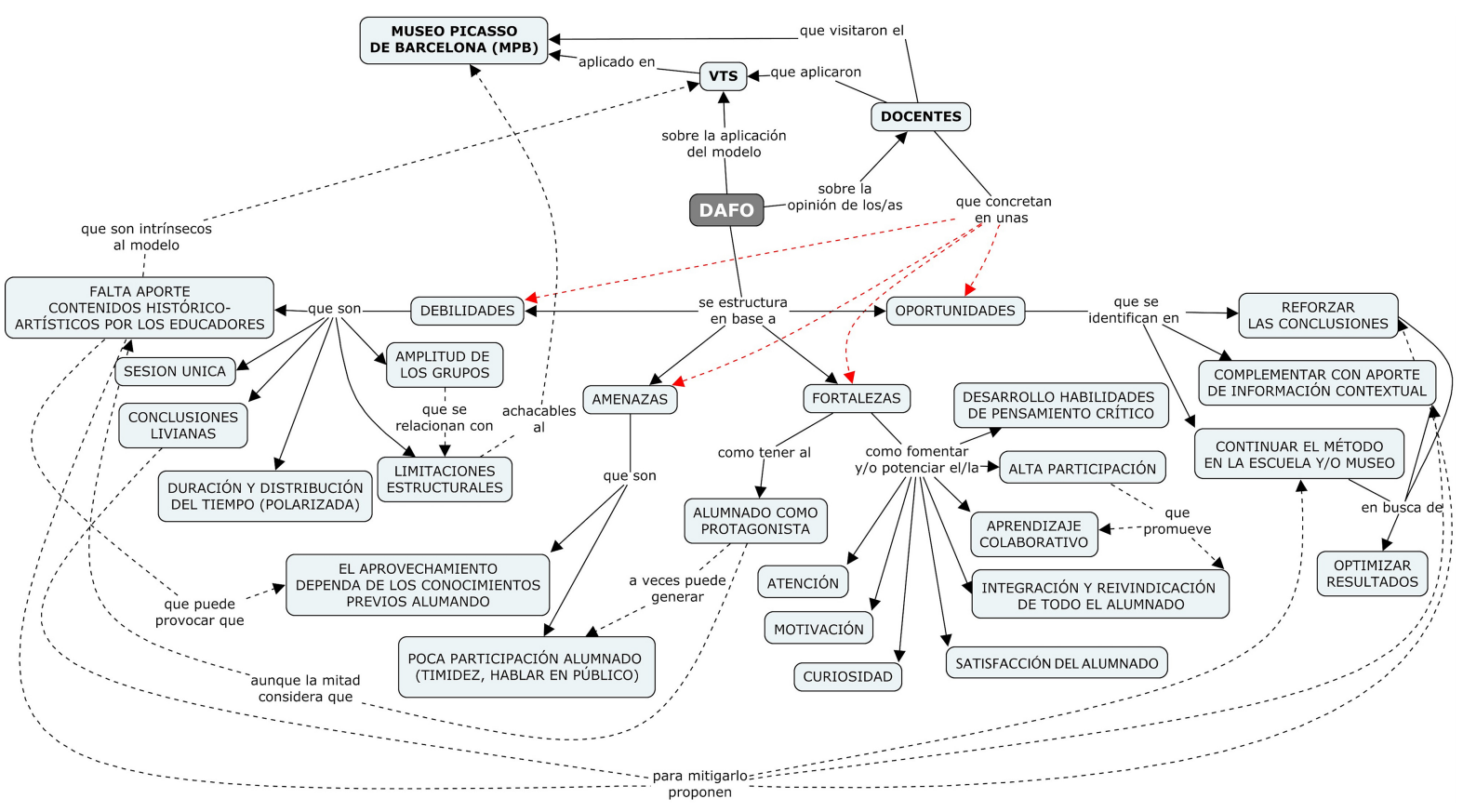

\section{Conclusiones}

Este estudio alcanza los objetivos planteados: analizar y articular las percepciones del profesorado de primaria sobre la adaptación del VTS a las actividades escolares del MPB. El establecimiento mediante un DAFO de las debilidades, amenazas, fortalezas y oportunidades que detectan y las relaciones -a menudo, tensiones-, entre los cuatro aspectos que muestra el mapa conceptual permiten hacer un diagnóstico y contribuir a su optimización.

Frente a las controversias suscitadas por el VTS, el DAFO evidencia que los docentes señalan muchos más aspectos positivos que negativos, predominando los primeros 
tanto en diversidad como en frecuencia. Detectan principalmente aspectos presentes e internos, si bien indican una gran variedad de oportunidades de mejora, a las que los investigadores suman las suyas.

Consideran fortalezas principales los efectos en el desarrollo de las habilidades ligadas al pensamiento crítico, las capacidades de expresión oral y la alfabetización visual de sus estudiantes, tres de las finalidades del VTS. Esto sitúa la investigación en la línea de las evaluaciones longitudinales de referencia (e.g. Adams et al, 2007; Pou, 2002; Tishman et al, 1999). Sin embargo, la mitad de los participantes subraya la importancia del factor tiempo en este método competencial (Kisida et al, 2016 y RA\&K, 2018), estableciendo como una debilidad principal su aplicación en una sola sesión en el museo. En consecuencia, se coincide con ellos en señalar como oportunidad continuar el trabajo en la escuela y/o en el museo, sugiriéndose que se amplíe la oferta de programas multivisita de mayor duración, en la estela del Thinking through art o el Projecte Tàndem del MPB con determinados centros escolares.

El segundo grupo de fortalezas gira alrededor del modelo educativo constructivista y significativo que propone el VTS; un modelo que definen como activo y centrado en el alumnado y que consideran que impulsa su protagonismo, curiosidad, motivación, atención y satisfacción. Estas opiniones rebaten la percepción del VTS como un método transmisivo y unidireccional camuflado en el que el educador sigue siendo el protagonista (e.g. Arriaga, 2010; López y Kivatinetz, 2006). No obstante, la mitad del profesorado destaca, como estas autoras, que no introducir contenidos históricoartísticos es una debilidad. Subscriben así las reticencias de sus colegas en estudios previos sobre la no adecuación a los contenidos y métodos del currículum escolar (e.g. Burchenal y Grohe, 2007; Cappello y Walker, 2016; Paunero, 2018), un hándicap de peso en un sistema obsesionado con contabilizar resultados (Hunter-Doniger y Herring, 2017).

Los investigadores coinciden con los docentes en que la implementación del método mejoraría al combinar las interpretaciones del alumnado con datos históricoartísticos, al creer con Arriaga (2010), Burnham y Kai-kee, (2011) y Hubard (2015) que aportar cierta cantidad de información contextual puede enriquecer el proceso interpretativo de los estudiantes sin coartar su libertad o su protagonismo. Esto reforzaría además indicadores y criterios de calidad sobre la diversidad de estrategias metodológicas que reiteran los últimos proyectos sobre evaluación de la acción educativa patrimonial (e.g. Gómez-Redondo et al, 2017; Comunitat d'Aprenenentatge Patrimoni $i$ Escola, 2018). Para ello se recomienda inspirarse en evoluciones del método como la del MoMA y en programas basados en las Thinking Routines de Project Zero (Visible Thinking, Artful Thinking, Slow Looking) que combinan contenidos del museo con los creados por sus usuarios (Ritchhart, 2007; Trouli, 2020).

El tercer conjunto de fortalezas se agrupa entorno al museo como espacio seguro (Ritchhart, 2007) para la participación y el debate, mostrando que parte del profesorado valora aspectos que conectan el VTS con el nuevo paradigma del museo como espacio de interacción (Simon, 2010) y con iniciativas basadas en metodologías dialógicas no predeterminadas (Hubard, 2015). Sorprendentemente, ninguno de los participantes hace mención de la ausencia de los discursos críticos y deconstructivos que defienden Arriaga (2010) y Mörsch (2009). De aquí que los investigadores reiteren 
con Huerta et al (2014) y Escribano-Miralles (2019) que, más allá de los debates académicos, es necesario consultar al profesorado para optimizar los programas escolares de los museos.

Este estudio no está exento de limitaciones. Pese a que los datos del CD y la ONP corresponden a 2017, y aunque no han podido estudiarse en relación a variables como los años de experiencia docente y las concepciones previas sobre arte y educación del profesorado, se abren prospectivas y se detectan temas, categorías y tensiones que permitirán actualizar el análisis en un futuro. El diagnóstico parcial se complementará próximamente al triangularlo con los del resto de agentes con el fin de mejorar la implementación del VTS en el MPB.

\section{Conflicto de intereses}

Los autores declaran no tener ningún conflicto de intereses.

\section{Agradecimientos}

Agradecemos sus aportaciones al profesorado participante, al Museo Picasso de Barcelona, a la Fundació la Caixa y a los expertos que han validado los instrumentos: Antonio Ruiz, Berta Palou, Ruth Vila, Montserrat Sampietro y Montserrat Morales. También a la Universidad de Barcelona, por la plaza predoctoral I0034899 concedida a la investigadora principal. Este proyecto ha recibido ayuda financiera de los gobiernos español y vasco a través de los grupos de investigación DIDPATRI, UB (2014SGR945) y GIPyPAC, UPV/EHU (IT1193-19).

\section{Referencias}

Adams, M., Foutz, S., Luke, J., y Stein, J. (2007). Thinking Through Art Isabella Stewart Gardner Museum School Partnership Program Year 3 Research Results. Institute for Learning Innovation.

Anderson, D., Kisiel, J. y Storksdieck, M. (2006). Understanding Teachers' Perspectives on Field Trips: Discovering Common Ground in Three Countries. Curator: The Museum Journal, 49(3), 365-386. https://doi.org/10.1111/j.21516952.2006.tb00229.x

Arriaga, A. (2010). Problemas y tendencias de interpretación de las obras de arte en las actividades educativas de museos. Revista Iberoamericana de Educación, 52(4), 1-12. https://doi.org/10.35362/rie5241788

Burchenal, M. y Grohe, M. (2007). Thinking through art: Transforming museum curriculum. Journal of Museum Education, 32(2), 111-122. https://doi.org/10.1080/10598650.2007.11510563

Burnham, R. y Kai-Kee, E. (2011). Teaching in the art museum: Interpretation as experience. Getty Publications

Cappello, M. y Walker, N. T. (2016). Visual Thinking Strategies: Teachers' Reflections on Closely Reading Complex Visual Texts Within the Disciplines. Reading Teacher, 70(3), 317-325. https://doi.org/10.1002/trtr.1523

Castejón, M. M. (2019). Hacia un museo social: estudio de una metodología participativa para vincular el Museo de Bellas Artes de Murcia a su territorio. (Tesis doctoral, Universidad

de

Murcia).

Digitum: 
https://digitum.um.es/digitum/bitstream/10201/73564/1/Mar\%C3\%ADa\%2 0Magdalena\%20Castejón\%20Ibáñez\%20Tesis\%20Doctoral.pdf

Comunitat de Pràctica Patrimoni i Escola (2018). Guia para evaluar el diseño de las actividades educativas patrimoniales. Col·lecció Museus 2039, Quaderns. Departament de Cultura. https://cultura.gencat.cat/web/.content/dgpc/museus/08.recursos/publicacions/quader ns/04_Guia-evaluar-diseno-actividades-educativas-patrimoniales.pdf

Dewhurst, M. y Hendrick, K. (2016). Dismantling Racism in Museum Education. Journal of Folklore and Education, 3, 25-30. https://locallearningnetwork.org/wpcontent/uploads/JFEv3.pdf

Escribano-Miralles, A. (2019). El Museo Arqueológico y la Enseñanza de la Historia en la Educación Formal. Una Mirada desde el Profesorado y los Educadores de Museo (tesis doctoral, Universidad de Murcia). Digitum http://hdl.handle.net/10201/86221

Falk, J. H. y Dierking, L. D. (2000). Learning from museums: Visitor experiences and the making of meaning. AltaMira Press.

Freire, P. (2005). Pedagogía del oprimido. Buenos Aires: Siglo XXI.

Gil-Zafra, M. A. (2001). Planificación estratégica: “Método DAFO". En Rodríguez Villasante, T., Montañés, M., y Martí, P. (Coords), Prácticas locales de creatividad social/1 (pp. 123-136). El Viejo Topo.

Gómez-redondo, C., Calaf, R. y Fontal, O. (2017). Diseño de un instrumento de análisis para recursos didácticos patrimoniales. Cadmo, 25(1), p. 63-80. http://dx.doi.org/10.3280/CAD2017-001008

González-Sanz, M., Feliu-Torruella, M. y Cardona-Gómez, G. (2017). Visual Thinking Strategies from the perspective of museum educators: a SWOT analysis of the practical implementation of the method. Revista de Educación, 375, 160-183. http://dx.doi.org/10.4438/1988-592X-RE-2016-375-339

Hailey, D., Miller, A. y Yenawine, P. (2015). Understanding Visual Literacy: The Visual Thinking Strategies Approach Abstract. En D. M. Baylen y A. D’Alba (Eds.), Essentials of Teaching and Integrating Visual and Media Literacy: Visualizing Learning, (pp. 219-236). Springer International. https://www.philipyenawine.com/teaching/2020/8/23/understandingvisual-literacy-the-visual-thinking-strategies-approach

Hein, G. (2006). Museum education. En Macdonald, S. (Ed.) A companion to museum studies (pp.340-352). Blackwell Publishing.

Hooper-Greenhill, E. (2000). Changing Values in the Art Museum: rethinking communication and learning. International Journal of Heritage Studies, 6(1), 931. https://doi.org/10.1080/135272500363715

Housen, A. (1999). Eye of the Beholder: Research, Theory and Practice. En Aesthetic and Art Education: A Transdisciplinary Approach. Fundación Calouste Gulbenkian. https://vtshome.org/wp-content/uploads/2016/08/5Eye-of-the-Beholder.pdf

Hubard, 0. (2015). Art museum education: Facilitating gallery experiences. Palgrave Macmillan.

Huerta, R., Vidagañ, M. y Munilla, G.. (2014). Percepciones del profesorado sobre la utilización del arte contemporáneo como herramienta pedagógica. Revista 
electrónica de investigación, docencia y creatividad, 3, 29-45. https://dialnet.unirioja.es/servlet/articulo?codigo $=6233445$

Huerta, R. (2009). Maestros y museos. Matrimonio de conveniencia. Revista Educación y Pedagogía, 21(55), 89-103.

https://revistas.udea.edu.co/index.php/revistaeyp/article/view/9759

Hunter-Doniger, T. y Herring, A. (2017) Artistic Realizations Found in Generalist Preservice Teachers' Courses: Rigor, Confidence, and Connections. Studies in Art Education, 58(4), 279-291, https://doi.org/10.1080/00393541.2017.1368289

Kisida, B., Bowen, D. H. y Greene, J. P. (2016). Measuring critical thinking: Results from an art museum field trip experiment. Journal of Research on Educational Effectiveness, 9(1), 171-187. https://doi.org/10.1080/19345747.2015.1086915

Kisida, B., Bowen, D. H. y Greene, J. P. (2017). Cultivating interest in art: Causal effects of arts exposure during early childhood. Early Childhood Research Quarterly, 45, 197-203. https://doi.org/10.1016/j.ecresq.2017.12.003

Lechuga-Jiménez, C. (2018). El museo y centros de arte contemporáneos como lugares de aprendizaje: VTS y la perspectiva de género. En B. Cantalapiedra,B., P. Aguilar, P. y P. Requeijo (Coords.), Fórmulas docentes de vanguardia (pp. 115-120). Gedisa.

Loran, M. (2014). Sortides a museus i espais patrimonials: procés de decisió, percepcions $i$ preferències de les escoles. Departament de Cultura. http://gabinet.dev.csuc.cat/bitstream/handle/20.500.12368/329/E150257.pd f?sequence $=1$ \&isAllowed $=y$

López, E. y Kivatinetz, M. (2006). Estrategias de pensamiento visual: ¿Método educativo innovador o efecto placebo para nuestros museos? Arte, Individuo y Sociedad, 18, 209-240.

https://revistas.ucm.es/index.php/ARIS/article/view/ARIS0606110209A

Luna, U. y Ibañez-Etxeberria, A. (2020). Cuando el museo se convirtió en espacio de aprendizaje. Educación y museos en Guipúzcoa en los años 80. Arte, Individuo y Sociedad 32(3), 445-462. https://doi.org/10.5209/aris.64869

Marcus, A. S., Levine, T. H., y Grenier, R. S. (2012). How secondary history teachers use and think about museums: Current practices and untapped promise for promoting historical understanding. Theory and Research in Social Education, 40(1), 66-97. https://doi.org/10.1080/00933104.2012.649466

Martín-Cáceres, M. J. y Cuenca-López, J. M. (2016). Communicating heritage in museums: outlook, strategies and challenges through a SWOT analysis. Museum Management and

Curatorship, 31(3),

299-316. https://doi.org/10.1080/09647775.2016.1173576

Meszaros, C. (2006). Now THAT is Evidence: Tracking Down the Evil "Whatever" Interpretation. Visitor studies today, 9(3), 10-15. https://www.informalscience.org/sites/default/files/VSA-a0a2r8-a 5730.pdf

Miles, M., Huberman, A. M. y Saldaña, J. (2014). Qualitative Data Analysis. A Methods Sourcebook. Sage.

Mörsch, C. (2009). At a Crossroads of Four Discourses: Documenta 12 Gallery Education in Between Affirmation, Reproduction, Deconstruction and Transformation. In Mörsch, C. (Ed.), Documenta 12, (pp. 9-31). IAE. 
https://www.academia.edu/27205348/At a Crossroads of Four Discourses d ocumenta 12 Gallery Education in between Affirmation Reproduction Decon struction and Transformation

Ordoñez, E. y Mohedano, I. (2019). El aprendizaje significativo como base de las metodologías innovadoras. Revista Educativa Hekademos, 26, 18-30. https://dialnet.unirioja.es/servlet/articulo?codigo $=6985274$

Paunero, D. (2018). Nuevos retos de interacción con el arte: las Estrategias de

Pensamiento Visual (VTS) en centros educativos de las islas Canarias. Her\&Mus. $\begin{array}{llll}\text { Heritage } \quad \text { M } & \text { Museography, 22-35. }\end{array}$ https://www.raco.cat/index.php/Hermus/article/view/338162.

Pou, C. (2002). Evaluación del programa Mira!. Un método interactivo de reflexión a través del arte. Laboratori de les arts (1998-2002). [Manuscrito sin publicar]. Fundació "La Caixa".

Ritchhart, R. (2007). Cultivating a culture of thinking in the museums. Journal of $\begin{array}{lll}\text { Museum } \quad \text { Education, 137-154. } & \text { 32(2), }\end{array}$ https://doi.org/10.1080/10598650.2007.11510565

RK\&A (2018). Impact study. The effects of facilitated single-visit art museum programs on students grades 4-6: Technical report. National Art Education Association \& Association of Art Museum Directors. https://www.arteducators.org/research/articles/377-naea-aamd-researchstudy-impact-of-art-museum-programs-on-k-12-students

Siddiqui, N., Gorard, S. y See, B. H. (2019). Can programmes like Philosophy for Children help schools to look beyond academic attainment? Educational Review, 71(2), 146-165. https://doi.org/10.1080/00131911.2017.1400948

Simon, N. (2010). The participatory museum. http://www.participatorymuseum.org/

Tishman, S., MacGillivray, D. y Palmer, P. (1999). Investigating the educational impact and potential of the Museum of Modern Art's Visual Thinking Curriculum: Final report. Harvard Project Zero. http://www.pz.harvard.edu/resources/investigating-the-educational-impactpotential-of-the-momas-visual-thinking-curriculum

Trouli, S. (2020). Museum education in Greece: histories, ideas and the realities behind the words we use. Icom education, 29, 115-134. http://ceca.mini.icom.museum/wpcontent/uploads/sites/5/2021/01/ICOMEducation29-compressé-aveccompression.pdf

Viladot, P. (2015). Motivacions, expectatives i objectius del professorat en les visites als museus de ciències (Tesis doctoral, Universitat de Barcelona). http://diposit.ub.edu/dspace/handle/2445/122115 
